\title{
Robust H-infinity State Estimation of Uncertain Neural Networks with Two Additive Time-Varying Delays
}

\author{
Xiaoping HU ${ }^{1}$, Yajun WANG, Jiakai DING and Dongming XIAO \\ Engineering Research Center of Advanced Mine Equipment, Ministry of Education, \\ Hunan University of Science and Technology, Xiangtan, 411201, China
}

\begin{abstract}
This study is mainly concerned with the problem of robust $H \infty$ state estimation of uncertain neural networks with two additive time-varying delays. A novel linear matrix inequalities (LMIs) is constructed based on Lyapunov-Krasovskii functionals ( $\mathrm{LKFs}$ ) which contains two additive time-varying delays components. LMIs method are used to estimate the derivative of LKFs, it is calculated that the derivative of the LKFs is smaller than zero, which proved that uncertain neural networks with two additive time-varying delays is globally asymptotically stable. Meantime, a stability criterion of error system is presented such that the $\mathrm{H} \infty$ performance is guaranteed. Finally, two numerical simulation examples have been performed to demonstrate the effectiveness of developed approach.
\end{abstract}

Keywords. linear matrix inequalities (LMIs), Ho state estimation, two time-varying delays, uncertain neural networks

\section{Introduction}

Time-varying delays has received much attention which exists in many industrial and engineering systems [1, 2]. Time-varying delays frequently cause oscillations, divergence or instability of the systems, so the systems stability is the main consideration for time-varying delay in many applications.

The stability problems for time-varying delays systems have been researched in recent years. For example, Wu [3] studied the stability of an uncertain systems, which contains multiple consecutive delay components. By considering the relationship between the time-varying delays and its upper bound, but there is no system state estimation, it is difficult to discover the change of the system, and the random interference items has not been added. Whether the delay is included according to the stability criteria, the delay systems is divided into two classes by Liu [4] which are delay-independent and delay-dependent. Two additive time-varying delays system is studied by Xiong [5] and establishes two novel integral inequalities, but the calculation process of this research is too cumbersome. The stability of continuous linear system with two additive time-varying delays is studied by $\mathrm{Xu}$ [6], which utilized the

\footnotetext{
${ }^{1}$ Corresponding author. Email: hxp210@163.com
} 
reciprocally convex combination technique. Subramanian [7] and Samidurai [8] studied robust analysis for uncertain neural networks with two additive time-varying delays by constructing Lyapunov-Krasovskii functions (LKFs) and making use of linear matrix inequalities (LMIs) technique. Liang [9] and Yuan [10] studied complex-valued neural networks (CVNNs) with two additive time-varying delays by constructing LKFs and making use of LMIs to solve the stable problems. The results show the convergence of the real and imaginary parts. The above literatures are for the stability analysis of two additive time-varying delays system. These literatures did not refer to the system by constructing state estimator.

Bao [11]. Hou [12], Liu[13], Shen [14], Zhang [15] and Zhao [16] studied with $H \infty$ state estimation problem for time-varying artificial neural networks. The aim of these papers is to design a time-varying $H \infty$ estimator, such that the dynamics of the estimation error satisfy the given $H \infty$ performance requirement. Liu [17] studied the problem of $H \infty$ state estimation of a static neural network with time-varying delay by constructing a suitable Lyapunov function and ensuring the attenuation of the results as early as possible, which indicates that the system is progressively stable under Lyapunov's conditions. $H \infty$ state estimation had been constructed in above literatures. Shao [18] studied an $H \infty$ control problem with delay-dependent stability condition, a new stability criteria is obtained, and finally the system is proved to be asymptotically stable by constructing a Lyapunov functional. Zhou [19] studied the robust $H \infty$ control problem for a delay singular system with parameter uncertainties. The influence of the system on the disturbance is attenuated, which indicate that the system is in the state of Lyapunov asymptotically stable by constructing a robust state feedback control law. A non-fragile state estimator of the recurrent delayed neural networks is designed by Yang [20] to ensure the existence of the desired estimators. The inadequacy of these researches is that they do not consider two additive time-varying delays issues. Among them, the $H \infty$ control essence is an optimization design using the $H \infty$ norm as the objective function. The $H \infty$ norm is a norm defined on the Hardy space. In the $H \infty$ control theory, it refers to the maximum singular value of the rational function matrix parsed in the right half plane of $S$.

Zhou [21] studied the problem of robust finite-time state estimation for a class of discrete-time neural networks with two delay components and Mrakovian jump parameters. And a new LKFs is constructed. Duan [22] investigated the state estimation for $H \infty$ control static neural network with two additive time delays. Time-varying delays often occur in engineering systems, network control, and biological network control. It is a factor that must be considered in the practice problems. In the problems of robust control and nonlinear asymptotically stable control, time-varying delays are factors that must be considered [23]. The difference between the leakage delays and the time-varying delays is that it will cause the instability of the system, and the time-varying delays can cause system delays [24].

There is less research in uncertain neural networks with two additive time-varying delays, in this paper, we investigate the $H \infty$ state estimation for uncertain neural networks with two additive time-varying delays. Based on LKFs method, a novel LMIs method has been established to ensure the global asymptotic stability of uncertain neural networks with two additive time-varying delays. Finally, two numerical simulation examples are used to illustrate the effectiveness of the proposed design method. 


\section{System model and Preliminaries}

Considering the following uncertain neural networks with two additive time-varying delays:

$$
\left\{\begin{array}{l}
\dot{x}(t)=-A_{1}(t) x(t)+A_{2}(t) f(\mathrm{x}(t))+A_{3}(t) x\left(t-\tau_{1}(t)-\tau_{2}(t)\right)+C_{1}(t) w(t)+u(t) \\
y(t)=B_{1}(t) x(t)+B_{2}(t) x\left(t-\tau_{1}(t)-\tau_{2}(t)\right)+D_{1}(t) w(t) \\
z(t)=H x(t) \\
x(t)=\varphi(t), t \in[-h, 0]
\end{array}\right.
$$

Where $x(t) \in\left[x_{1}(t), x_{2}(t), \cdots, x_{n}(t)\right]^{T} \in \mathfrak{R}^{n}$ is the state vector, $y(t)$ is the measurement, $u(t)$ is the control input, $z(t) \in \square$ to be estimated is a linear combination of the state, $w(t)$ is disturbance input and $w(t) \in L^{2}[0, \infty]$, and $A_{1}, A_{2}, A_{3}, C_{1}, B_{1}, B_{2}, D_{1}$ and $H$ are known real constant matrices, $f(x(t))=\left[f_{1}\left(x_{1}(t)\right), f_{2}\left(x_{2}(t)\right) \ldots \ldots f_{n}\left(x_{n}(t)\right)\right]^{T}$ denotes the neuron activation function and a constant input vector, and. $A_{1}(t)=A_{1}+\Delta A_{1}, A_{2}(t)=A_{2}+\Delta A_{2}, A_{3}(t)=A_{3}+\Delta A_{3}$, $B_{1}(t)=B_{1}+\Delta B_{1}, B_{2}(t)=B_{2}+\Delta B_{2}, C_{1}(t)=C_{1}+\Delta C_{1}, D_{1}(t)=D_{1}+\Delta D_{1}, \Delta A_{1}, \Delta A_{2}, \Delta A_{3}, \Delta B_{1}, \Delta B_{2}, \Delta C_{1}$ and $\Delta D_{1}$ is real matrix. These parameters represent the uncertainty of the system. $\varphi(t)$ is represents a given initial condition.

Assumption 1. The parameter uncertainties $\Delta A_{1}, \Delta A_{2}, \Delta A_{3}, \Delta B_{1}, \Delta B_{2}, \Delta C_{1}$ and $\Delta D_{1}$ are of the form:

$$
\left[\begin{array}{lllllll}
\Delta A_{1} & \Delta A_{2} & \Delta A_{3} & \Delta B_{1} & \Delta B_{2} & \Delta C_{1} & \Delta D_{1}
\end{array}\right]=H F(t)\left[\begin{array}{lllllll}
M_{11} & M_{12} & M_{21} & M_{22} & S_{1} & S_{2} & S_{3}
\end{array}\right]
$$

Among these parameters, $H, M_{11}, M_{12}, M_{21}, M_{22}, S_{1}, S_{2}, P_{1}$ is known real matrix of appropriate dimension. $F(t) \in R^{i \times j}, i>0, j>0$ is the real unknown time-varying matrix. So,

$$
F(t)^{T} F(t) \leq I, \forall t \geq 0
$$

Assumption 2. The time-varying delays $\tau_{1}(t), \tau_{2}(t)$ satisfy

$$
\begin{gathered}
0 \leq \tau_{1}(t) \leq \tau_{1} \leq \infty, 0 \leq \tau_{2}(t) \leq \tau_{2} \leq \infty \\
\tau_{1}(t) \leq \mu_{1}, \tau_{2}(t) \leq \mu_{2} \\
\tau=\tau_{1}+\tau_{2}, \mu=\mu_{1}+\mu_{2}
\end{gathered}
$$

where $\tau_{1}, \tau_{2}$ are positive constants.

Assumption 3 [25]. Each neuron activation function $f_{i}(),. i=1,2, \ldots, n$ satisfies the following condition:

$$
0 \leq \frac{f_{i}(\alpha)-f_{i}(\beta)}{\alpha-\beta} \leq l_{i}, \forall \alpha, \beta \in R, \alpha \neq \beta
$$

where $l_{i}, i=1,2, \ldots, n$ are constants, and diagonal matrix $L=\operatorname{diag}\left\{l_{i}\right\}$. Then, constructing a state estimator for estimation of $Z(t)$ as follows 


$$
\left\{\begin{aligned}
\dot{x}(t) & =-A_{1}(t) \hat{x}(t)+A_{2}(t) f(\hat{x}(t))+A_{3}(t) \hat{x}\left(t-\tau_{1}(t)-\tau_{2}(t)\right) \\
& +K\left(y(t)-B_{1}(t) \hat{x}(t)+B_{2}(t) \hat{x}\left(t-\tau_{1}(t)-\tau_{2}(t)\right)\right)+u \\
\hat{z}(t)=H \hat{x}(t) & \\
x(t) & =0, t \in[-h, 0]
\end{aligned}\right.
$$

where $\hat{x}(t) \in \square^{n}$ denotes the estimated state, $\hat{z}(t) \in \square^{n}$ denotes the estimated measurement of $z(t)$, and $K$ is the state estimator gain matrix.

Denoting the errors by $e(t)=x(t)-\hat{x}(t)$ and $\quad \tau(t)=\tau_{1}(t)+\tau_{2}(t) \quad, \quad \bar{z}(t)=z(t)-\hat{z}(t)$, $e\left(t-\tau_{1}(t)-\tau_{2}(t)\right)=x\left(t-\tau_{1}(t)-\tau_{2}(t)\right)-\hat{x}\left(t-\tau_{1}(t)-\tau_{2}(t)\right)$

Then, based on (1) and (8), the error system of the form is obtained:

$$
\left\{\begin{aligned}
\dot{e}(t) & =-\left(A_{1}(t)+K B_{1}(t)\right) e(t)+A_{2}(t) g(e(t))+\left(A_{3}(t)-K B_{2}(t)\right) e\left(t-\tau_{1}(t)-\tau_{2}(t)\right) \\
& +\left(C_{1}(t)-K D_{1}(t)\right) w(t) \\
\bar{z}(t) & =H e(t)
\end{aligned}\right.
$$

Where $\quad g(e(t))=f(x(t))-f(\hat{x}(t))$,

$$
\left\{\begin{aligned}
\dot{e}(t) & =-\left(A_{1}(t)+K B_{1}(t)\right) e(t)+A_{2}(t) g(e(t))+\left(A_{3}(t)-K B_{2}(t)\right) e(t-\tau(t)) \\
& +\left(C_{1}(t)-K D_{1}(t)\right) w(t) \\
\bar{z}(t) & =H e(t)
\end{aligned}\right.
$$

In this paper we will study the stability of (10) so that guaranteed its $H \infty$ performance. Moreover, it is proved by numerical simulation that the state estimation of the system error equation tends to zero, which proves that the system is asymptotically stable.

Definition 1 [25]. Given a prescribed level of noise attenuation $\gamma \geq 0$, a proper state estimator (8) is founded, the equilibrium point of the result error system (10) with $w(t)=0$ is globally asymptotically stable, and

$$
\|\bar{z}(t)\|_{2}<\gamma\|w(t)\|_{2}
$$

under zero-initial conditions for all nonzero $w(t) \in L_{2}[0, \infty)$, where

$$
\|x(t)\|_{2} \square \sqrt{\int_{0}^{\infty} x^{T}(t) x(t) d t}
$$

In this case, error system (10) is globally asymptotically stable with $H \propto$ performance $\gamma$.

\section{Results and Proof}

In this section, the global asymptotic stability of the model (10) with the initial condition (2) (7) is discussed and the main results are given as follows:

Theorem 1. Considering the uncertain neural networks with two additive time-varying delays (1), If there is an appropriate dimension of the matrix $Y$, for given 
scalars $h_{1}, h_{2}>0$, let $\gamma$ to be a prescribed constant, then the guaranteed $H \infty$ performance state estimation problem is solvable, if there exist real matrices $Q_{1}, Q_{2}, Q_{3}, Q_{4}, Q_{5}, Q_{6}>0$ $T>0, P_{1}>0, R_{i}>0, S_{i}>0(i=1,2,3), M_{j e}>0(j=1,2, e=1,2)$; positive diagonal matrices $P_{2}=\operatorname{diag}\left\{\eta_{i}\right\}$, so the following LMIs are satisfied:

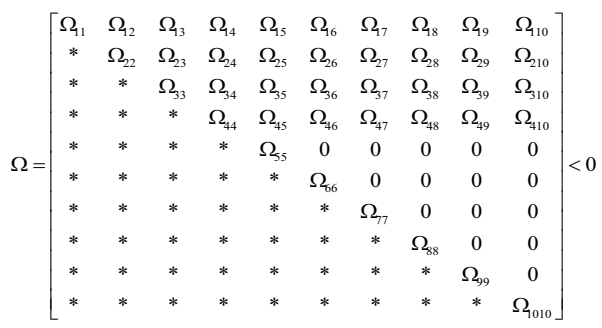

where

$$
\begin{aligned}
& \Omega_{11}=-A_{1} P_{1}+B_{1} Y-A_{1}^{T} P_{1}^{T}+B_{1}^{T} Y^{T}+Q_{1}+Q_{2}+Q_{3}+Q_{4}+Q_{5}+Q_{6}+M_{11}^{T}+M_{11}+M_{12}^{T}+M_{12}-T+H^{T} H, \Omega_{1010}=-\gamma^{2} I \\
& \Omega_{12}=A_{3} P_{1}-B_{2} Y-A_{1} P_{1}^{T}+B_{1}^{T} Y^{T}+R_{2}-T-M_{11}{ }^{T}+M_{21}, \Omega_{13}=A_{2} P_{1}-A_{1}^{T} P_{1}^{T}+B_{1}^{T} Y^{T}-M_{12}{ }^{T}+M_{22}, \Omega_{14}=R_{2}, \\
& \Omega_{15}=A_{2} P_{2}-A_{1}{ }_{1}^{T} P_{1}^{T}+B_{1}^{T} Y^{T}, \Omega_{16}=\tau_{1}\left(A_{3}^{T} P_{1}^{T}+B_{1}^{T} Y^{T}\right)-A_{1}^{T} P_{1}^{T}+B_{1}{ }^{T} Y^{T}, \Omega_{17}=\tau_{2}\left(A_{3}{ }^{T} P_{1}^{T}+B_{1}^{T} Y^{T}\right)-A_{1}{ }^{T} P_{1}^{T}+B_{1}^{T} Y^{T}, \Omega_{18}=\tau_{1} S_{1} \\
& \Omega_{19}=\tau_{2} S_{2}, \Omega_{110}=P_{1} C_{1}, \Omega_{22}=A_{1} P_{1}-(1-\tau) M_{11}{ }^{T}+(1-\tau) M_{22}, \Omega_{23}=0, \Omega_{24}=R_{2}-T, \Omega_{25}=\left(A_{1} P_{1}\right)^{T}, \Omega_{26}=\tau_{1}\left(A_{1} P_{1}\right)^{T}, \\
& \Omega_{27}=\tau_{2}\left(A_{1} P_{1}\right)^{T}, \Omega_{28}=\tau_{2} S_{3}, \Omega_{29}=0, \Omega_{210}=0, \Omega_{33}=-Q_{2}-(1-\tau) M_{11}{ }^{T}+(1-\tau) M_{22}, \Omega_{34}=0, \Omega_{35}=\left(B_{1} P_{1}\right)^{T}, \\
& \Omega_{36}=\tau_{1}\left(B_{1} P_{1}\right)^{T}, \Omega_{37}=\tau_{2}\left(B_{1} P_{1}\right)^{T}, \Omega_{38}=0, \Omega_{39}=\tau_{2} R_{3}, \Omega_{310}=0, \Omega_{44}=-P_{1}, \Omega_{45}=B_{1}{ }^{T} \Omega_{46}=B_{2}{ }^{T}, \Omega_{47}=\tau_{2} B_{1}{ }^{T}, \\
& \Omega_{48}=\tau_{2} B_{2}{ }^{T}, \Omega_{49}=0, \Omega_{410}=D_{1}, \Omega_{55}=-\tau R_{3}, \Omega_{66}=-\tau_{1} R_{1}, \Omega_{77}=-\tau_{2} R_{2}, \Omega_{88}=-\tau_{1} R_{1}^{T}, \Omega_{99}=-\tau_{2} R_{2}{ }^{T},
\end{aligned}
$$

where $K=P^{-1} Y$

Proof 1. constructing the following Lyapunov-Krasovskii functional:

$$
\begin{gathered}
V(t)=V_{1}(t)+V_{2}(t)+V_{3}(t)+V_{4}(t) \\
V_{1}\left(e_{t}\right)=e^{T}(t) P_{1} e(t)+2 \sum_{i=1}^{n} P \int_{0}^{e_{i}(t)} g(s) d s \\
V_{2}\left(e_{t}\right)=\int_{t-\tau_{1}(t)}^{t} e^{T}(s) Q_{1} e(s) d s+\int_{t-\tau_{2}(t)}^{t} e^{T}(s) Q_{2} e(s) d s+\int_{t-\tau(t)}^{t} e^{T}(s) Q_{3} e(s) d s \\
+\int_{t-\tau_{1}}^{t} e^{T}(s) Q_{4} e(s) d s+\int_{t-\tau_{2}}^{t} e^{T}(s) Q_{5} e(s) d s+\int_{t-\tau}^{t} e^{T}(s) Q_{6} e(s) d s \\
V_{3}(e)=\int_{t-\tau(t)}^{t}\left[\begin{array}{c}
e(s) \\
g(e(s))
\end{array}\right]^{T}\left[\begin{array}{cc}
R_{1} & R_{2} \\
* & R_{3}
\end{array}\right]\left[\begin{array}{c}
e(s) \\
g(e(s))
\end{array}\right] d s, \\
V_{4}\left(e_{t}\right)=\tau_{1} \int_{-\tau_{1}}^{0} \int_{t+\theta}^{t} e^{T}(s) R_{1} e(s) d s d \theta+\tau \int_{-\tau}^{0} \int_{t+\theta}^{t} e^{T}(s) R_{2} e(s) d s d \theta+\tau_{2} \int_{-\tau_{2}}^{0} \int_{t+\theta}^{t} e^{T}(s) R_{3} e(s) d s d \theta
\end{gathered}
$$

Taking the time-derivative of ${ }^{V(t)}$ along the trajectories of yields. Robust stabilization of the system. In this case, error system (10) is globally asymptotically stable with $H \infty$ performance $\gamma$. 


$$
\begin{aligned}
\dot{V}_{1}(e(t)) & =2 e^{T}(t) P_{1} \dot{e}(t)+2 g^{T}(\mathrm{e}(t)) P_{2} \dot{e}(t) \\
& =2 e^{T}(t) P_{1}\left[-\left(A_{1}(t)+K B_{1}(t)\right) e(t)+A_{2}(t) g(e(t))+\left(A_{3}(t)-K B_{2}(t)\right) e(t-\tau(t))\right. \\
& \left.+\left(C_{1}(t)-K D_{1}(t)\right) w(t)\right]+2 g^{T}(\mathrm{e}(t)) P_{2}\left[-\left(A_{1}(t)+K B_{1}(t)\right) e(t)+A_{2}(t) g(e(t))\right. \\
& \left.+\left(A_{3}(t)-K B_{2}(t)\right) e(t-\tau(t))+\left(C_{1}(t)-K D_{1}(t)\right) w(t)\right]
\end{aligned}
$$

$$
\begin{aligned}
= & -2 e^{T}(t) P_{1}\left(A_{1}(t)+K B_{1}(t)\right) e(t)+2 e^{T}(t) P_{1}\left(A_{3}(t)-K B_{2}(t)\right) e(t-\tau(t))+2 e^{T}(t) P_{1} A_{2}(t) g(e(t)) \\
& +2 e^{T}(t) P_{1}\left(C_{1}(t)-K D_{1}(t)\right) w(t)-2 g^{T}(\mathrm{e}(t)) P_{2}\left(A_{1}(t)+K B_{1}(t)\right) e(t)+2 g^{T}(\mathrm{e}(t)) P_{2}\left(A_{3}(t)-K B_{2}(t)\right) e(t-\tau(t)) \\
& +2 g^{T}(\mathrm{e}(t)) P_{2} A_{2}(t) g(e(t))+2 g^{T}(\mathrm{e}(t)) P_{2}\left(C_{1}(t)-K D_{1}(t)\right) w(t)
\end{aligned}
$$

$$
\begin{aligned}
& \dot{V}_{2}(e(t)) \leq e^{T}(t)\left(Q_{1}+Q_{2}+Q_{3}+Q_{4}+Q_{5}+Q_{6}\right) e(t)-e^{T}\left(t-\tau_{1}\right) Q_{1} e\left(t-\tau_{1}\right) \\
& -e^{T}\left(t-\tau_{2}\right) Q_{2} e\left(t-\tau_{2}\right)-e^{T}(t-\tau) Q_{3} e(t-\tau)-\left(1-\mu_{1}\right) e^{T}\left(t-\tau_{1}(t)\right) Q_{4} e\left(t-\tau_{1}(t)\right) \\
& -\left(1-\mu_{2}\right) e^{T}\left(t-\tau_{2}(t)\right) Q_{5} e\left(t-\tau_{2}(t)\right)-(1-\mu) e^{T}(t-\tau(t)) Q_{6} e(t-\tau(t)) \\
& \dot{V}_{3}(e(t))=\left[\begin{array}{c}
e(t) \\
g(e(t))
\end{array}\right]^{T}\left[\begin{array}{cc}
R_{1} & R_{2} \\
* & R_{3}
\end{array}\right]\left[\begin{array}{c}
e(t) \\
g(e(t))
\end{array}\right]-(1-\dot{\tau}(t))\left[\begin{array}{c}
e(t-\tau(t)) \\
g(e(t-\tau(t)))
\end{array}\right]^{T}\left[\begin{array}{cc}
R_{1} & R_{2} \\
* & R_{3}
\end{array}\right]\left[\begin{array}{c}
e(t-\tau(t)) \\
g(e(t-\tau(t)))
\end{array}\right] \\
& \leq e^{T}(t) R_{1} e(t)+g^{T}(e(t)) R_{3} g\left(e(t)+2 e^{T}(t) R_{2} g(e(t))-(1-\mu) e^{T}(t-\tau(t)) R_{1} e(t-\tau(t))\right. \\
& -(1-\mu) g^{T}\left(\mathrm{e}(t-\tau(t)) R_{3} g(\mathrm{e}(t-\tau(t)))-2(1-\mu) e^{T}(t-\tau(t)) R_{2} g(\mathrm{e}(t-\tau(t)))\right. \\
& \dot{V}_{4}(e(t))=\tau_{1}^{2} \dot{e}^{\mathrm{T}}(t) R_{1} \dot{e}(t)-\tau_{1} \int_{t-\tau_{1}}^{t} \dot{e}^{\mathrm{T}}(s) R_{1} \dot{e}(s) d s+\tau_{2}^{2} \dot{e}^{T}(t) R_{3} \dot{e}(t)-\tau_{2} \int_{t-\tau_{2}}^{t} \dot{e}^{T}(s) R_{3} \dot{e}(s) d s \\
& +\tau^{2} \dot{e}^{T}(t) R_{2} \dot{e}(t)-\tau \int_{t-\tau}^{t} \dot{e}^{T}(s) R_{2} \dot{e}(s) d s
\end{aligned}
$$

Among the equation of the $\dot{V}_{4}(e(t))$, Then $\dot{V}_{4}(e(t))$ is obtained through above formulas:

$$
\begin{aligned}
\dot{V}_{4}(e(t)) & \leq \tau_{1}^{2} \dot{e}^{T}(t) R_{1} \dot{e}(t)-\tau_{1} \int_{t-\tau_{1}}^{t} \dot{e}^{T}(s) R_{1} \dot{e}(s) d s+\tau_{2}^{2} \dot{e}^{T}(t) R_{3} \dot{e}(t)-\tau_{2} \int_{t-\tau_{2}}^{t} \dot{e}^{T}(s) R_{3} \dot{e}(s) d s \\
& +\tau^{2} \dot{e}^{T}(t) R_{2} \dot{e}(t)-\tau \int_{t-\tau}^{t} \dot{e}^{T}(s) R_{2} \dot{e}(s) d s \\
& =\tau^{2} \dot{e}^{T}(t) R_{2} \dot{e}(t)+\alpha^{T}(t)\left[\begin{array}{cc}
R_{2} & T \\
* & R_{2}
\end{array}\right] \alpha(t)+\tau_{2}^{2} \dot{e}^{T}(t) R_{3} \dot{e}(t)+\alpha^{T}(t)\left[\begin{array}{cc}
R_{3} & T \\
* & R_{3}
\end{array}\right] \alpha(t) \\
& +\tau_{1}^{2} \dot{e}^{T}(t) R_{1} \dot{e}(t)+\alpha^{T}(t)\left[\begin{array}{cc}
R_{1} & T \\
* & R_{1}
\end{array}\right] \alpha(t) \\
& =\xi^{T}(t) \Sigma_{1} \xi(t)+\xi^{T}(t) \Sigma_{2} \xi(t)+\xi^{T}(t) \Sigma_{3} \xi(t)=\xi^{T}(t)\left(\Sigma_{1}+\Sigma_{1}+\Sigma_{3}\right) \xi(t) \\
& \leq \xi^{T}(t) \Sigma_{1} \xi(t)
\end{aligned}
$$

$$
\Sigma=\left[\begin{array}{cccccccccc}
\Sigma_{11} & \Sigma_{12} & \Sigma_{13} & \Sigma_{14} & \Sigma_{15} & \Sigma_{16} & \Sigma_{17} & \Sigma_{18} & \Sigma_{19} & \Sigma_{110} \\
* & \Sigma_{22} & \Sigma_{23} & \Sigma_{24} & \Sigma_{25} & \Sigma_{26} & \Sigma_{27} & \Sigma_{28} & \Sigma_{29} & \Sigma_{210} \\
* & * & \Sigma_{33} & \Sigma_{34} & \Sigma_{35} & \Sigma_{36} & \Sigma_{37} & \Sigma_{38} & \Sigma_{39} & \Sigma_{310} \\
* & * & * & \Sigma_{44} & \Sigma_{45} & \Sigma_{46} & \Sigma_{47} & \Sigma_{48} & \Sigma_{49} & \Sigma_{410} \\
* & * & * & * & \Sigma_{55} & 0 & 0 & 0 & 0 & 0 \\
* & * & * & * & * & \Sigma_{66} & 0 & 0 & 0 & 0 \\
* & * & * & * & * & * & \Sigma_{77} & 0 & 0 & 0 \\
* & * & * & * & * & * & * & \Sigma_{88} & 0 & 0 \\
* & * & * & * & * & * & * & * & \Sigma_{99} & 0 \\
* & * & * & * & * & * & * & * & * & \Sigma_{1010}
\end{array}\right]<0
$$

\section{Where}

$$
\begin{aligned}
& \Sigma_{11}=-A_{1} P_{1}+B_{1} Y-A_{1}^{T} P_{1}^{T}+B_{1}^{T} Y^{T}+Q_{1}+Q_{2}+Q_{3}+Q_{4}+Q_{5}+Q_{6}+M_{11}^{T}+M_{11}+M_{12}^{T}+M_{12}-T+H^{T} H \\
& \Sigma_{12}=A_{3} P_{1}-K B_{2} Y-A_{1}^{T} P_{1}^{T}+B_{1}^{T} Y^{T}+R_{2}-T-M_{11}^{T}+M_{21}
\end{aligned}
$$




$$
\begin{aligned}
& \Sigma_{13}=A_{2} P_{1}-A_{1}^{T} P_{1}^{T}+B_{1}^{T} Y^{T}-M_{12}{ }^{T}+M_{22} \quad, \quad \Sigma_{14}=R_{2} \quad, \quad \Sigma_{15}=\left(A_{3} P_{1}+B_{1} Y\right)^{T}-A_{1}^{T} P_{1}^{T}+B_{1}^{T} Y^{T} \quad, \\
& \Sigma_{16}=\tau_{1}\left(A_{3} P_{1}+B_{1} Y\right)^{T}-A_{1}^{T} P_{1}^{T}+B_{1}^{T} Y^{T} \quad \Sigma_{17}=\tau_{2}\left(A_{3} P_{1}+B_{1} Y\right)^{T}-A_{1}^{T} P_{1}^{T}+B_{1}^{T} Y^{T}, \Sigma_{18}=\tau_{1} S_{1} \quad \Sigma_{19}=\tau_{2} S_{2}, \quad \Sigma_{110}=P_{1} C_{1}, \\
& \Sigma_{22}=A_{1} P_{1}-(1-\tau) M_{11}{ }^{T}+(1-\tau) M_{22}, \quad \Sigma_{23}=0, \quad \Sigma_{24}=R_{2}-T \quad \Sigma_{25}=\left(A_{1} P_{1}\right)^{T}, \quad \Sigma_{26}=\tau_{1}\left(A_{1} P_{1}\right)^{T} \quad \Sigma_{27}=\tau_{2}\left(A_{1} P_{1}\right)^{T}, \\
& \Sigma_{28}=\tau_{2} S_{3} \quad, \quad \Sigma_{29}=0 \quad, \quad \Sigma_{210}=0 \quad, \quad \Sigma_{33}=-Q_{2}-(1-\tau) M_{22}{ }^{T}-(1-\tau) M_{22} \quad, \quad \Sigma_{34}=0 \quad, \quad \Sigma_{35}=\left(B_{1} P_{1}\right)^{T} \\
& \Sigma_{36}=\tau_{1}\left(B_{1} P_{1}\right)^{T}, \Sigma_{37}=\tau_{2}\left(B_{1} P_{1}\right)^{T}, \Sigma_{38}=0, \Sigma_{39}=\tau_{2} R_{3}, \Sigma_{310}=0, \Sigma_{44}=-P_{1}, \Sigma_{45}=B_{1}^{T}, \Sigma_{46}=B_{2}{ }^{T}, \Sigma_{47}=\tau_{2} B_{1}^{T}, \\
& \Sigma_{48}=\tau_{2} B_{2}{ }^{T}, \quad \Sigma_{49}=0, \Sigma_{410}=D_{1}, \quad \Sigma_{55}=-\tau R_{3} \quad \Sigma_{66}=-\tau_{1} R_{1}, \quad \Sigma_{77}=-\tau_{2} R_{2}, \quad \Sigma_{88}=-\tau_{1} R_{1}^{T}, \quad \Sigma_{99}=-\tau_{2} R_{2}{ }^{T} \text {, } \\
& \Sigma_{1010}=-\gamma^{2} I \\
& \alpha(t)=\left[\alpha_{1}^{T}(t), \alpha_{2}^{T}(t), \alpha_{3}^{T}(t), \alpha_{4}^{T}(t)\right]^{T} \\
& \alpha_{1}(t)=e(t-\tau(t))-e(t-\tau), \alpha_{2}(t)=e(t-\tau(t))-e(t-\tau)-\frac{2}{t-\tau(t)} \int_{t-\tau}^{t-\tau(t)} e(s) d s \\
& \alpha_{3}(t)=e(t)-e(t-\tau(t)), \alpha_{4}(t)=e(t)-e(t-\tau(t))-\frac{2}{\tau(t)} \int_{t-\tau(t)}^{t} e(s) d s \\
& \xi(t)=\left[e^{T}(t), e^{T}(t-\tau(t)), e^{T}(t-\tau), e^{T}\left(t-\tau_{1}(t)\right), e^{T}\left(t-\tau_{1}\right), e^{T}\left(t-\tau_{2}(t)\right), e^{T}\left(t-\tau_{2}\right), g^{T}(e(t)), \hat{x}(t-\tau(t)), w^{T}(t)\right]^{T}
\end{aligned}
$$

Combining the above results, $\dot{V}(e(t))$ can be obtained:

$$
\begin{aligned}
\dot{V}(e(t)) \leq & -e^{T}(t)\left[P_{1}\left(A_{1}(t)+K B_{1}(t)\right)+\left(A_{1}(t)+K B_{1}(t)\right)^{T} P_{1}\right] e(t)+2 e^{T}(t) P_{1}\left(A_{3}(t)-K B_{2}(t)\right) e\left(t-\tau_{1}(t)-\tau_{2}(t)\right) \\
& +2 e^{T}(t) P_{1} A_{2}(t) g(e(t))+2 e^{T}(t) P_{1}(C(t)-K D(t)) w(t)+2 g^{T}\left(e_{i}(t)\right) \Lambda \dot{e}(t)+e^{T}(t)\left(Q_{1}+Q_{2}+Q_{3}\right) e(t) \\
& -e^{T}\left(t-\tau_{1}\right) Q_{1} e\left(t-\tau_{1}\right)-e^{T}\left(t-\tau_{2}\right) Q_{2} e\left(t-\tau_{2}\right)-e^{T}(t-\tau) Q_{3} e(t-\tau)-\left(1-\mu_{1}\right) e^{T}\left(t-\tau_{1}(t)\right) Q_{1} e\left(t-\tau_{1}(t)\right) \\
& -\left(1-\mu_{2}\right) e^{T}\left(t-\tau_{2}(t)\right) Q_{2} e\left(t-\tau_{2}(t)\right)-(1-\mu) e^{T}(t-\tau(t)) Q_{3} e(t-\tau(t))+e^{T}(t) R_{1} e(t)+g^{T}(e(s)) R_{3} g(e(s)) \\
& +2 e^{T}(t) R_{2} g(e(s))-(1-\mu) e^{T}(t-\tau(t)) R_{1} e(t-\tau(t))-(1-\mu) g^{T}(e(t-\tau(t))) R_{3} g(e(t-\tau(t))) \\
& -2(1-\mu) e^{T}(t-\tau(t)) R_{2} g(e(t-\tau(t)))+\xi^{T}(t)\left(\Sigma_{1}+\Sigma_{2}+\Sigma_{3}\right) \xi(t) \\
& \leq \xi^{T}(t)\left(\Sigma_{1}+\Sigma_{2}+\Sigma_{3}\right) \xi(t) \\
& \leq \xi^{T}(t) \Sigma \xi(t)<0
\end{aligned}
$$

Therefore, if LMIs is to be work, then $\dot{V}(e(t))<0$. The neural networks (1) is asymptotically stable. This completes the Proof 1 .

Since the function $f(x(t))$ satisfy (7). Then, for any $e(t) \neq 0$.

$$
0 \leq \frac{g_{i}(e(t), \hat{x}(t))}{e(t)}=\frac{f(x(t))-f(\hat{x}(t))}{x(t)-\hat{x}(t)} \leq l_{i}
$$

Under the zero-initial condition, it is obvious that $\left.V(e(t))\right|_{t=0}=0$. For dealing easily. Let

$$
J_{\infty}=\int_{0}^{t}\left[\bar{z}^{T}(s) \bar{z}(s)-\gamma^{2} w^{T}(s) w(s)\right] d s, t>0
$$

Then,

$$
J_{\infty} \leq \int_{0}^{t}\left[\bar{z}^{T}(s) \bar{z}(s)-\gamma^{2} w^{T}(s) w(s)\right] d s+V(e(t))-\left.V(e(t))\right|_{t=0}, t>0
$$

Then for any $w(t) \in L^{2}[0, \infty]$,

$$
J_{\infty} \leq \int_{0}^{t} p\left[\bar{z}^{T}(s) \bar{z}(s)-\gamma^{2} w^{T}(s) w(s)+V(e(\dot{s}))\right] d s
$$


Based on the equalities and inequalities, we can deduce that:

$$
\bar{z}^{T}(t) \bar{z}(t)-w^{T}(t) w(t)+\dot{V}(e(t)) \leq \xi^{T}(t)\left[\Sigma_{1}+\tau^{2} \Sigma_{2}^{T} R_{2} \Sigma_{2}\right] \xi(t)
$$

So if $\Sigma_{1}+\tau^{2} \Sigma_{2}^{T} R_{2} \Sigma_{2}<0$, then there must exist a sufficiently small scalar $\sigma$, so $\Sigma_{1}+\tau^{2} \Sigma_{2}{ }^{T} R_{2} \Sigma_{2}+\sigma I \leq 0$. Then, it is easy to obtain

that for any $w(t) \neq 0$.

$$
\begin{aligned}
\bar{z}^{T}(t) \bar{z}(t)-w^{T}(t) w(t)+\dot{V}(e(t)) & \leq \xi^{T}(t)\left[\Sigma_{1}+\tau^{2} \Sigma_{2}{ }^{T} R_{2} \Sigma_{2}\right] \xi(t) \\
& \leq-\sigma \xi^{T}(t) \xi(t) \leq-\sigma w^{T}(t) w(t)<0
\end{aligned}
$$

Proof 2. The system is robustly, asymptotically stable if the following inequalities is satisfied:

$$
\Omega+\varphi_{1} F(t) \varphi_{2}^{T}+\varphi_{2} F(t) \varphi_{1}^{T}+\varphi_{3} F(t) \varphi_{4}^{T}+\varphi_{4} F(t) \varphi_{3}^{T}+\varphi_{5} F(t) \varphi_{6}^{T}+\varphi_{6} F(t) \varphi_{5}^{T}<0
$$

Where $\varphi_{1}=\left[\begin{array}{llllllllll}H^{T} P_{1} & 0 & 0 & 0 & 0 & 0 & 0 & 0 & 0 & 0\end{array}\right]^{T}, \varphi_{2}=\left[\begin{array}{llllllllll}0 & 0 & 0 & 0 & 0 & 0 & 0 & 0 & 0 & H^{T} R_{3}\end{array}\right]^{T}$, $\varphi_{3}=\left[\begin{array}{llllllllll}M_{11} & 0 & M_{12} & 0 & 0 & 0 & 0 & 0 & 0 & 0\end{array}\right]^{T}, \varphi_{4}=\left[\begin{array}{llllllllll}M_{21} & 0 & M_{22} & 0 & 0 & 0 & 0 & 0 & 0 & 0\end{array}\right]^{T}$, $\varphi_{5}=\left[\begin{array}{llllllllll}S_{1} & 0 & S_{2} & 0 & 0 & 0 & 0 & 0 & 0 & 0\end{array}\right]^{T}, \quad \varphi_{6}=\left[\begin{array}{llllllllll}S_{3} & 0 & 0 & 0 & 0 & 0 & 0 & 0 & 0 & 0\end{array}\right]^{T}$

If (13) is satisfied, then the following inequalities:

$\Omega+\varepsilon_{1}^{-1} \varphi_{1} \varphi_{1}^{T}+\varepsilon_{1} \varphi_{2} \varphi_{2}^{T}+\varepsilon_{2}^{-1} \varphi_{3} \varphi_{3}^{T}+\varepsilon_{2} \varphi_{4} \varphi_{4}^{T}+\varepsilon_{3}^{-1} \varphi_{5} \varphi_{5}^{T}+\varepsilon_{3} \varphi_{6} \varphi_{6}^{T} \equiv \Omega+\psi$

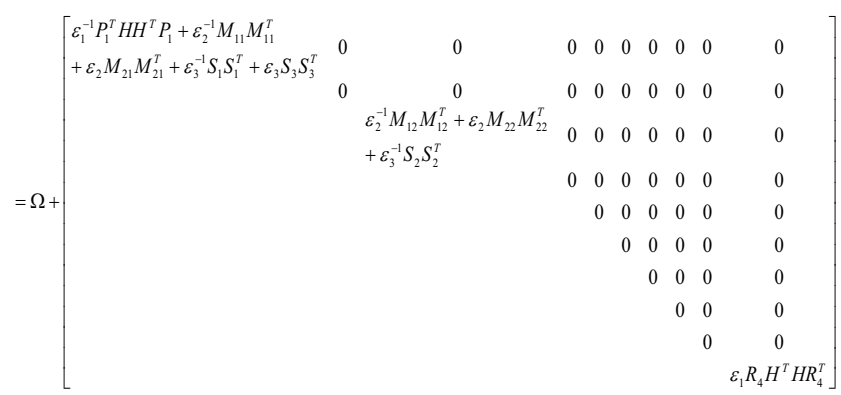

where $\varepsilon_{1}>0, \varepsilon_{2}>0, \varepsilon_{3}>0$.

Then, the inequalities given in (33) is equivalent to the LMIs (25). Thus, if the LMIs given in (25) is satisfied, then the system (10) is robust asymptotically stable. This completes the Proof 2.

Corollary 1 Considering the neural networks with two additive time-varying delays system (1), for given scalars $\mu<1$ and $K$, let $\gamma$ be a prescribed constant, the guaranteed $H \infty$ performance state estimation problem is solvable if there exist real matrices $Y>0, M_{j e}>0(j=1,2, e=1,2)$; and diagonal matrices $P_{2}=\operatorname{diag}\left\{\eta_{i}\right\}$ with appropriate dimensions, then the following LMIs are satisfied: 


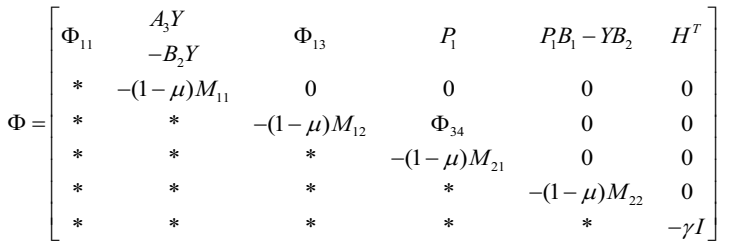

$\Phi_{11}=-P_{1} A_{1}-A_{1}^{T} P_{1}^{T}-B_{1} Y-Y^{T} B_{1}^{T}+M_{11}+M_{12}+M_{21}+M_{22}$

$\Phi_{13}=-(1-\mu)\left(M_{11}+M_{12}\right)-2 P_{2}, \Phi_{34}=-(1-\mu)\left(M_{21}+M_{22}\right)-2 P_{2}$

Moreover, the gain matrix $K$ of the state estimator of (8) can be designed as $K=P_{1}^{-1} Y$.

Remark 1. Theorem 1 provides a novel robust $H \infty$ state estimation of uncertain neural networks stability criterion for system (1) with two additive time-varying delays components, it has been verified by a form of less complex LMIs.

Remark 2. Based on the Lyapunov stability criterion, a novel LMIs is constructed to prove that the derivative of the LKFs is smaller than zero, and that the system (1) is asymptotically stable. table 1 .

Remark 3. The state estimation uncertain neural networks methods are listed in

Table 1. state estimation of uncertain neural network methods.

\begin{tabular}{cccccccc}
\hline numbers & 1 & 2 & 3 & 4 & 5 & 6 & 7 \\
\hline \multirow{4}{*}{ methods } & $H \infty$ & Robust & State & Extended & Delay & Non-fragile & Recursive \\
& State & finite-time & estimation & dissipative & dependent & state & state \\
& estimation & state & {$[26]$} & state & state & estimation & estimation \\
& {$[22]$} & estimation & & estimation & estimation & {$[29]$} & {$[30]$} \\
& & {$[21]$} & & {$[27]$} & {$[28]$} & &
\end{tabular}

Compared with other theories, the $H \infty$ theory can give delay-dependent criteria, so that the error system has globally asymptotic stability $H \infty$ performance.

Remark 4. The structure of paper is organized as follows: In Section 2, problem model are given. In Section 3, a new

theorem and three corollaries are established. In Section 4, two simulation results are provided to demonstrate the effectiveness of the developed approach. Finally, Section 5 summarizes this work.

\section{Numerical Examples}

Two numerical simulation examples are to be presented to show the feasibility of the developed approach.

Example1. Considering the system (10) with following parameters:

$A_{1}=\left[\begin{array}{cc}-1 & 0 \\ -1 & -1\end{array}\right], A_{2}=\left[\begin{array}{cc}-1 & 0 \\ -1 & -1\end{array}\right], A_{3}=\left[\begin{array}{cc}-2 & 0 \\ 0 & -1\end{array}\right], B_{1}=\left[\begin{array}{cc}0.1 & 0 \\ 0 & 0.1\end{array}\right], \quad B_{2}=\left[\begin{array}{l}0 \\ 1\end{array}\right], \quad C=\left[\begin{array}{ll}1 & 0 \\ 0 & 1\end{array}\right], \quad D=\left[\begin{array}{ll}1 & 0 \\ 0 & 1\end{array}\right], x_{\text {delay }}=\left[\begin{array}{lll}0 & 0 & 0.2\end{array}\right]$, $x=\left[\begin{array}{c}0.2 \\ 0.3 \\ 1\end{array}\right], \tau_{1}=0.15, \tau_{2}=0.24, t=0, d t=0.001$. 
In addition, the activation function is chosen as $f(x)=\tanh (x)$, the time-varying delay by $\tau(t)=0$. It is easy to get $K=\left[\begin{array}{ll}-4.0107 & -1.7896 \\ -1.7896 & -4.6767\end{array}\right], \tau=0.39$.the noise disturbance is assumed be $w(t)=\frac{1}{1.2 t+0.8}$.

$P_{1}=\left[\begin{array}{cc}0.6749 & -0.0197 \\ -0.0197 & 0.5245\end{array}\right], \quad P_{2}=\left[\begin{array}{cc}0.1972 & 0 \\ 0 & 0.1972\end{array}\right], \quad Y=\left[\begin{array}{cc}-4.0107 & -1.7896 \\ -1.7896 & -4.6767\end{array}\right], \quad S_{1}=\left[\begin{array}{cc}-2.8839 & 0.0969 \\ 0.0969 & -2.4532\end{array}\right], \quad S_{2}=\left[\begin{array}{cc}-0.0163 & 0.0291 \\ 0.0291 & 0.0200\end{array}\right]$, $S_{3}=\left[\begin{array}{cc}0.0250 & -0.0874 \\ -0.0874 & -0.0408\end{array}\right], Q_{1}=\left[\begin{array}{cc}581.417 & 58.2298 \\ 58.2298 & -436.2641\end{array}\right]$,

$Q_{2}=\left[\begin{array}{ll}0.8732 & 0.1111 \\ 0.1111 & 1.0659\end{array}\right], Q_{3}=Q_{4}=Q_{5}=Q_{6}=\left[\begin{array}{cc}-436.4433 & -57.6238 \\ -57.6238 & 64.8827\end{array}\right], M_{11}=\left[\begin{array}{cc}282.9611 & 44.2305 \\ 44.2305 & 37.1869\end{array}\right], M_{12}=\left[\begin{array}{cc}300.9950 & 43.6859 \\ 43.6859 & 53.3194\end{array}\right]$, $M_{21}=\left[\begin{array}{cc}280.9950 & 42.1068 \\ 42.1068 & 33.6494\end{array}\right], M_{22}=\left[\begin{array}{cc}300.6336 & 43.8862 \\ 43.8862 & 53.7267\end{array}\right]$

With the option $H \infty$ performance index $\gamma=1.3811$. Fig 1 shows the responses of the estimation error curves which generated by random initial value, it confirms the feasibility of the developed LMIs method through the designed state estimator of uncertain neural networks with two additive time-varying delays.

By applying the MATLAB LMI toolbox, it is found that LMIs (13) is feasible. As Fig.1 illustrates, the initial matrix of the example1 is a two-dimensional matrix, there are four estimation error curves. The state estimation tends to 0 rapidly. Therefore, it is proved that uncertain neural networks with two additive time-varying delays is globally asymptotically stable through proposed LMIs. The calculated minimum $H \infty$ performance index $\gamma$ with different $\mu<1$ values are listed in Table 2. One can know clearly that the results obtained by Corollary 1 can provide smaller $H \infty$ performance index $\gamma$ than recently existing method in [1].

Table 2. Minimum $H \infty$ performance index $\gamma$ with different $\mu$.

\begin{tabular}{cccc}
\hline$\mu$ & 0.4 & 0.5 & 0.7 \\
\hline Reference [1] & 0.4632 & 0.5301 & 1.3819 \\
Corollary 1 & 0.0035 & 0.1544 & 0.4402 \\
\hline
\end{tabular}

Example2. Considering a delayed neural network of Corollary 1 with following parameters:

$$
A_{1}=\left[\begin{array}{ccc}
-1 & 1 & 0 \\
2 & 1 & -1 \\
1 & -1 & 2
\end{array}\right], B_{1}=\left[\begin{array}{ccc}
-1 & 1 & 0 \\
-1 & 1 & -1 \\
-1 & -1 & 1
\end{array}\right], B_{2}=\left[\begin{array}{ccc}
-1 & 0 & 0 \\
0 & 1 & -1 \\
-1 & 0 & 1
\end{array}\right], A_{3}=\left[\begin{array}{ccc}
-1 & 0 & 0 \\
1 & 1 & 0 \\
1 & -1 & -1
\end{array}\right], I=\left[\begin{array}{ccc}
1 & 0 & 0 \\
0 & 1 & 0 \\
0 & 0 & 1
\end{array}\right], \gamma=0.5, \mu=0.5
$$

In addition, the activation function is chosen as $f(x)=\tanh (x)$, the time-varying delay by $\tau(t)=0$. It is easy to get



$$
\begin{aligned}
& Y=10^{-9} *\left[\begin{array}{ccc}
0.0120 & 0.0394 & 0.0110 \\
0.0394 & 0.2970 & -0.1812 \\
0.0110 & -0.1812 & 0.2188
\end{array}\right], H=10^{-11} *\left[\begin{array}{ccc}
0.6127 & 0 & 0 \\
0 & 0.6127 & 0 \\
0 & 0 & 0.6127
\end{array}\right], M_{11}=10^{-9} *\left[\begin{array}{ccc}
0.0244 & -0.0197 & -0.0132 \\
-0.0197 & 0.0939 & -0.1353 \\
-0.0132 & -0.1353 & 0.1524
\end{array}\right] \text {, } \\
& M_{12}=10^{-9} *\left[\begin{array}{ccc}
0.0400 & 0.0266 & -0.0520 \\
0.0266 & 0.1108 & -0.1033 \\
-0.0520 & -0.1033 & 0.1156
\end{array}\right], M_{21}=10^{-9} *\left[\begin{array}{ccc}
0.0727 & 0.0332 & -0.0076 \\
0.0332 & 0.1481 & -0.0368 \\
-0.0076 & -0.0368 & 0.1161
\end{array}\right], M_{22}=10^{-10 *}\left[\begin{array}{ccc}
0.1040 & -0.1395 & -0.1606 \\
-0.1395 & -0.3238 & 0.0840 \\
-0.1606 & 0.0840 & -0.0893
\end{array}\right] \text {. }
\end{aligned}
$$






Figure 1. Estimation errors $e_{1}, e_{2}, e_{3}$ and $e_{4}$

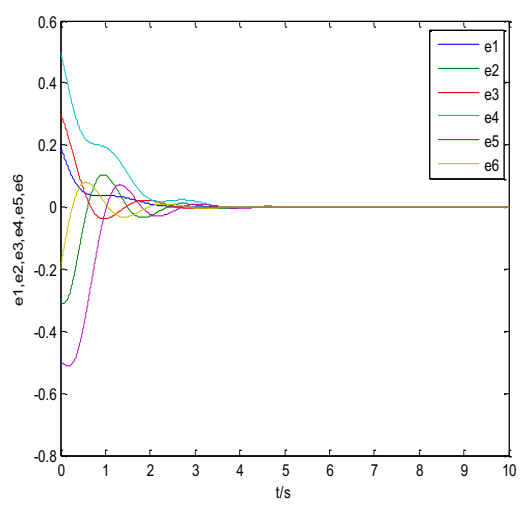

Figure 2. Estimation errors $e_{1}, e_{2}, e_{3}, e_{4}, e_{5}$ and $e_{6}$

The global asymptotically stable simulation results of the system (10) with the above parameters is illustrated in Fig.2.

the initial matrix of the example 2 is a three-dimensional matrix, there are six estimation error curves.

\section{Conclusions}

In this paper, the problem of $H \infty$ state estimation of uncertain neural networks with two additive time-varying delays has been studied. Based on LKFs method, a novel LMIs method has been established to ensure the global asymptotic stability of uncertain neural networks with two additive time-varying delays. It has fine convergence speed through constructed LMIs. Two numerical simulation examples have been performed to demonstrate the feasibility of the developed approach.

We would like to point out that this work did not include the $H \infty$ state estimation of uncertain neural networks with leaking time delay.

\section{Acknowledgment}

This work is supported by National Natural Science Foundation of China (No. 51875195) and National Natural Science Foundation of China (No. 61572185).

\section{References}

[1] S. Arik, "Global asymptotic stability of a class of dynamical neural networks," IEEE Transactions on circuits and systems I: Fundamental theory and applications, vol. 47, no. 4, pp. 568-571, 2000.

[2] D. Xiao, X. Li, and K. He, "Power Balance of Starting Process for Pipe Belt Conveyor Based on Master-Slave Control," IEEE Access, vol. 6, pp. 16924-16931, 2018.

[3] H. Wu, X. Liao, W. Feng, S. Guo, and W. Zhang, "Robust stability analysis of uncertain systems with two additive time-varying delay components," Applied Mathematical Modelling, vol. 33, no. 12, pp. 4345-4353, 2009.

[4] Y. Liu, S.-M. Lee, and H. Lee, "Robust delay-depent stability criteria for uncertain neural networks with two additive time-varying delay components," Neurocomputing, vol. 151, pp. 770-775, 2015.

[5] L. Xiong, J. Cheng, J. Cao, and Z. Liu, "Novel inequality with application to improve the stability criterion for dynamical systems with two additive time-varying delays," Applied Mathematics and Computation, vol. 321, pp. 672-688, 2018. 
[6] H.-T. Xu, C.-K. Zhang, L. Jiang, and J. Smith, "Stability analysis of linear systems with two additive time-varying delays via delay-product-type Lyapunov functional," Applied Mathematical Modelling, vol. 45, pp. 955-964, 2017.

[7] K. Subramanian, P. Muthukumar, and S. Lakshmanan, "Robust stabilization of uncertain neural networks with additive time-varying delays*," IFAC-PapersOnLine, vol. 49, no. 1, pp. 154-159, 2016.

[8] R. Samidurai and R. Sriraman, "Robust dissipativity analysis for uncertain neural networks with additive time-varying delays and general activation functions," Mathematics and Computers in Simulation, vol. 155, pp. 201-216, 2019.

[9] J. Liang, K. Li, Q. Song, Z. Zhao, Y. Liu, and F. E. Alsaadi, "State estimation of complex-valued neural networks with two additive time-varying delays," Neurocomputing, 2018.

[10] Y. Yuan, Q. Song, Y. Liu, and F. E. Alsaadi, "Synchronization of complex-valued neural networks with mixed two additive time-varying delays," Neurocomputing, 2018.

[11] H. Bao, J. Cao, J. Kurths, A. Alsaedi, and B. Ahmad, "H$\infty$ state estimation of stochastic memristor-based neural networks with time-varying delays," Neural Networks, vol. 99, pp. 79-91, 2018.

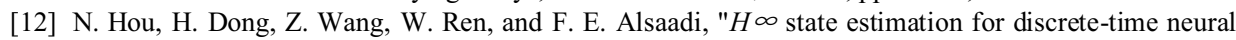
networks with distributed delays and randomly occurring uncertainties through fading channels," Neural Networks, vol. 89, pp. 61-73, 2017.

[13] B. Liu, X. Ma, and X.-C. Jia, "Further results on $\mathrm{H} \infty$ state estimation of static neural networks with time-varying delay," Neurocomputing, vol. 285, pp. 133-140, 2018.

[14] H. Shen, M. Xing, S. Huo, Z.-G. Wu, and J. H. Park, "Finite-time $H \infty$ asynchronous state estimation for discrete-time fuzzy Markov jump neural networks with uncertain measurements," Fuzzy Sets and Systems, vol. 356, pp. 113-128, 2019.

[15] S. Zhang, D. Ding, G. Wei, Y. Liu, and F. E. Alsaadi, " $H \infty$ state estimation for artificial neural networks over redundant channels," Neurocomputing, vol. 226, pp. 117-125, 2017.

[16] Z. Zhao, Z. Wang, L. Zou, and H. Liu, "Finite-horizon $H \infty$ state estimation for artificial neural networks with component-based distributed delays and stochastic protocol," Neurocomputing, vol. 321, pp. 169-177, 2018.

[17] Y. Liu, S.-M. Lee, O. Kwon, and J. H. Park, "A study on $H \infty$ state estimation of static neural networks with time-varying delays," Applied Mathematics and Computation, vol. 226, pp. 589-597, 2014.

[18] H. Shao, X. Zhu, Z. Zhang, and G. Zhong, "Delay-dependent $H \infty$ control for systems with two additive time-vary delays," Journal of Control Engineering and Technology, vol. 3, no. 2, pp. 90-97, 2013.

[19] S. Zhou and W. X. Zheng, "Robust $H \infty$ control of delayed singular systems with linear fractional parametric uncertainties," Journal of the Franklin Institute, vol. 346, no. 2, pp. 147-158, 2009.

[20] F. Yang, H. Dong, Z. Wang, W. Ren, and F. E. Alsaadi, "A new approach to non-fragile state estimation for continuous neural networks with time-delays," Neurocomputing, vol. 197, pp. 205-211, 2016.

[21] X. Zhou and Q. Zhu, "Robust finite-time state estimation for uncertain discrete-time Markovian jump neural networks with two delay components," Neurocomputing, vol. 283, pp. 64-72, 2018.

[22] Q. Duan, H. Su, and Z.-G. Wu, "Hळ state estimation of static neural networks with time-varying delay," Neurocomputing, vol. 97, pp. 16-21, 2012.

[23] R. Saravanakumar, M. S. Ali, J. Cao, and H. He, "Ho state estimation of generalised neural networks with interval time-varying delays," International Journal of Systems Science, vol. 47, no. 16, pp. 3888-3899, 2016.

[24] H. Zhu, R. Rakkiyappan, and X. Li, "Delayed state-feedback control for stabilization of neural networks with leakage delay," Neural Networks, vol. 105, pp. 249-255, 2018.

[25] H. He, F. Gang, and J. Cao, "Guaranteed performance state estimation of static neural networks with time-varying delay," Neurocomputing, vol. 74, no. 4, pp. 606-616, 2011.

[26] L. Qian, Q. Zhu, S. Zhong, X. Wang, and J. Cheng, "State estimation for uncertain Markovian jump neural networks with mixed delays," Neurocomputing, vol. 182, no. C, pp. 82-93, 2016.

[27] Q. Li, Q. Zhu, S. Zhong, and F. Zhong, "Extended dissipative state estimation for uncertain discrete-time Markov jump neural networks with mixed time delays," Isa Transactions, vol. 66, pp. 200-208, 2017.

[28] H. J. Yu, H. Yong, and W. Min, "Delay-Dependent State Estimation for Neural Networks with Time-Varying Delay," Neurocomputing, vol. 275, p. S0925231217315448, 2018.

[29] R. Li, J. Cao, A. Alsaedi, and T. Hayat, "Non-fragile state observation for delayed memristive neural networks," Neurocomputing, vol. 245, no. C, pp. 102-113, 2017.

[30] J. Hu, Z. Wang, S. Liu, and H. Gao, "A variance-constrained approach to recursive state estimation for time-varying complex networks with missing measurements," Automatica, vol. 64, no. C, pp. 155-162, 2016. 